\title{
Improving the MRI Tumor Segmentation Process Using Appropriate Image Processing Techniques
}

\author{
Ahmed Basil Al-Othman \\ Faculty of Computing, Universiti Teknologi Malaysia \\ E-mail: ahmedbasil0081@yahoo.com \\ Zohair Al-Ameen ${ }^{1,2}$ \\ ${ }^{1}$ Faculty of Computing, Universiti Teknologi Malaysia \\ ${ }^{2}$ Faculty of Information Sciences and Engineering, Management and Science University \\ E-mail: qizohair3@live.utm.my \\ Ghazali Bin Sulong \\ Faculty of Computing, Universiti Teknologi Malaysia \\ E-mail: ghazali@spaceutm.edu.my
}

\begin{abstract}
Segmenting tumor from MRI images is an essential but time consuming manual duty. Performing an automatic segmentation is a defying task since different forms of tumor tissue exist for diverse patients and in many cases the tumor is similar to the normal tissue. Various studies proposed earlier to handle the issue of precisely segmenting the tumor but they discard the degradations and their effect to the precision of the segmentation. This article provides a more precise segmentation process through the use of appropriate preprocessing algorithms. The authors studied many enhancement and restoration algorithms and selected the NL-means, Laplacian filter and histogram equalization to be used as preprocessing techniques. Experimental results showed that using a suitable preprocessing scheme would produce a better segmentation process.
\end{abstract}

Index Terms - Medical image processing, Tumor segmentation, MRI images, Non-local Means, Histogram equalization, Laplacian filter.

\section{INTRODUCTION}

A tumor is a mass of tissue that grows out of control of the normal forces that regulates growth. Brain tumor has a higher mortality rate when compared to more common types of cancer. This underlines the need of accurate prognosis of brain tumor in health care industry [1]. MRI has become a widely-used method of high-quality medical imaging, especially in brain imaging [2]. Image segmentation refers to the process of partitioning a digital image into multiple segments, also known as super pixels. Image segmentation is an important process in computer vision and image processing, which divides an image into a number of disjoint regions such that the pixels have high similarity in order to use its different fields; science, medical, agriculture and industrial fields [3]. Besides, Magnetic Resonance Imaging (MRI) images are known to be corrupted by noise [4], blur [5] and low contrast artifact [6]. Therefore, this article discusses the issue of achieving a better segmentation through the use of proper image restoration and enhancement techniques. Moreover, the accurate and automatic segmentation of a brain tumor on MRI image is of great interest for assessing tumor growth and treatment responses, enhancing computerassisted surgery, and constructing tumor growth models [7]. For those reasons, an automatic and reliable tumor segmentation method would be a useful tool since the medical image segmentation plays an instrumental role in clinical diagnosis [8]. The rest of the article sections are organized as the following: in section two, a brief elucidation about some related studies is presented as the used literature review. Moreover, detailed explanations about the employed algorithms are delivered in section three as a methodology. Also, the results of the proposed methodology are illustrated in section four as the experimental results. Likewise, a detailed discussion about the use of certain algorithms and its results is deliberated in section five. Finally, a brief closure about this article is provided in section six as a conclusion.

\section{LITERATURE REVIEW}

In this section, three recent studies are briefly described. The first study was presented by [9] which proposed a texture based analysis to identify irregularity in the brain and automatic segmentation using seeded region growing segmentation. The method of tumor detection based on the texture of the MRI is proposed to segment the abnormality from the regular adjacent, although small quantities misclassified, but the segmentation result is precise. Moreover, [10] suggested a strong technique for automatic detection of tumor for brain MRI images. He also proposed a new morphological technique which is used for the stripped skull region from the brain images. Detecting tumor precisely based on seeded region growing segmentation on an improved single seed point selection algorithm is 
applied to detect the tumor. The proposed model consists of three major parts pre-processing, segmentation using region growing, and post-processing. Finally, the study provided by [11] combined seeding region growing algorithm with fuzzy k-means clustering, and precision algorithm to measure gray and white matter tissues. The proposed algorithm is to segment gray matter/white matter in magnetic resonance image (MRI). The algorithm contains three procedures: fuzzy K-means, region growing, performance measure procedure for integrating regions and taking out the final segmentation the experimental results show that the proposed technique produces perfect and stable results.

\section{METHODOLOGY}

The proposed methodology consists mainly of a preprocessing and a segmentation phase. The first phase involves three primary techniques that are: image denoising using a non-local means algorithm, image enhancement using a histogram equalization algorithm and image sharpening using a Laplacian filter. The second phase involves a reign growing segmentation algorithm. The outcome obtained from these two phases is considered as the final results as shown in Figure 1.

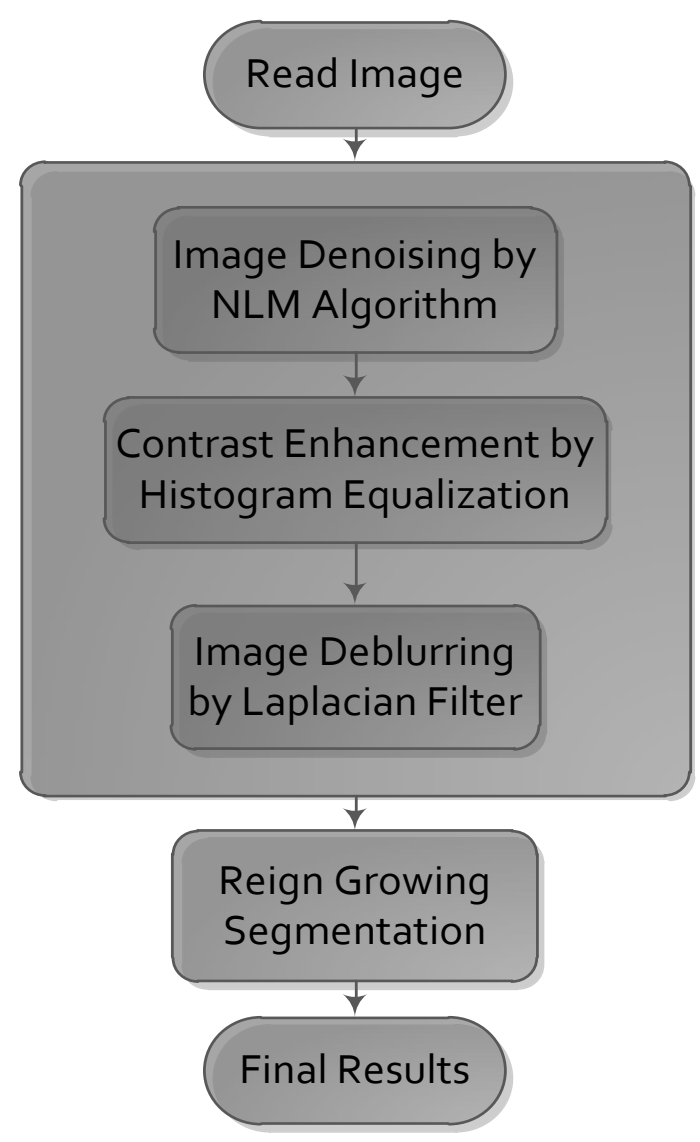

Figure 1 . The procedural scheme of the proposed methodology

\section{A. Non-local Means}

In any image, a pixel may have similarity with its neighboring pixels which is introduced as a self-similarity concept [12]. The non-local means algorithm works based on self-similarity concepts. Figure 2 describes where the self-similarity can be found in an image. Suppose three windows of pixels denoted by $p, q 1$ and $q 2$. The pixel $p$ is more similar to $q 1$ rather than $q 2$. Therefore, adjacent or non-adjacent pixels will have similar neighboring pixels [13]. As displayed in Figure 2, the pixels on top or bottom of $p$ tend to have similar intensities. Hence, the selfsimilarity concept is used to determine the denoised pixels.

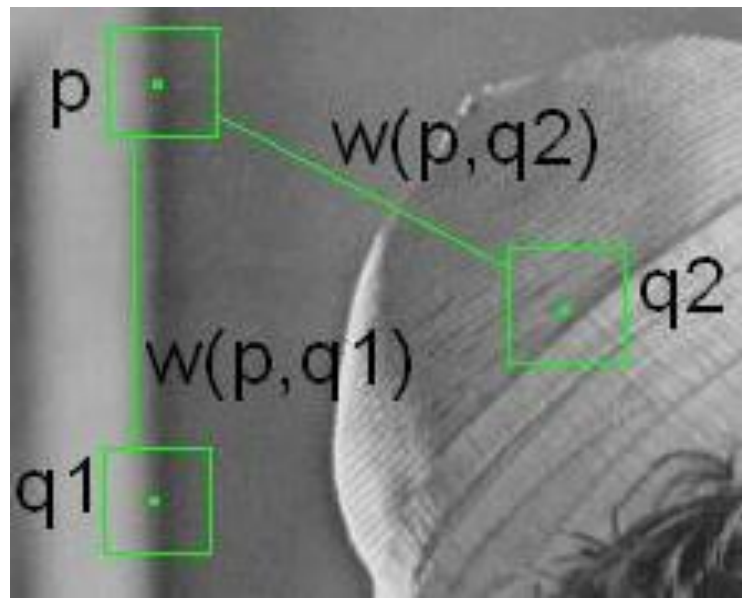

Figure 2. The self-similarity concept

The non-local means denoising algorithm functions as the following: Firstly, assuming that $v(j)$ is a noisy pixels, its corresponding weights $w(i, j)$ are calculated by evaluating the neighborhood of pixels $i$ and $j$ using the following formula:

$$
N L(v)(i)=\sum_{j \in I} w(i, j) v(j)
$$

The weights $w(i, j)$ should fulfill such conditions:

$$
0 \leq w(i, j) \leq 1
$$

$$
\sum_{i} w(i, j) ? ?
$$

Secondly, calculate the neighborhood by taking pixel $i$ as the center and Rsim as a predefined radius, and the squared neighboring pixels of $(i)$ are denoted by $\left(N_{i}\right)$. Then, employ a Gaussian weighted Euclidean distance to calculate the similarity between $(\mathrm{Ni})$ and $v(\mathrm{Nj})$ as the following [13] [14]: 
24 Improving the MRI Tumor Segmentation Process Using Appropriate Image Processing Techniques

$$
E\|v(N i)-v(N j)\|_{2, a}^{2}=\|u(N i)-u(N j)\|_{2, a}^{2}+2 \sigma
$$


Where, common values for $\operatorname{Rsim}$ are 3 and 4 to give neighborhoods of size $7 \times 7$ and 9x9. Finally, the weights are calculated using the subsequent formula:

$w(i, j)=\frac{1}{Z(i)} e^{-\frac{\|v(N i)-v(N j)\|_{2, a}^{2}}{h^{2}}}$

Where, $Z(I)$ is the contrast and it used to normalize the weight in the range between $[1,0]$. The contrast $Z(i)$ can be computed using the following equation:

$Z(i)=\sum_{j} e^{-\frac{\|v(N i)-v(N j)\|_{2, a}^{2}}{h^{2}}}$

Where, $(h)$ is weight-decay control parameter. The control parameter $(h)$ can be set based on the standard deviation of white noise $(\sigma)$ in the image, such that $(h)$ should be set between $10 \sigma$ and $15 \sigma$ [13] [14].

\section{B. Histogram Equalization}

The histogram equalization is one of the famous methods used to enhance the contrast of medical images [15] [16]. Its basic idea lies in mapping the gray levels based on the probability distribution of these inputs gray levels. Likewise, It flattens and stretches the dynamic range of the image's histogram, resulting in overall contrast enhancement [17] [18]. Moreover, it became a popular method due to its simplicity, efficiency and rapidity [19] [20]. The histogram equalization works as the following: Let $I$ and $O$ are the boundaries of the images, the image levels defined by $\left[l s, l_{\mathrm{f}}\right]$, be initialized as $[0, L-1]$. Then, calculate the image histogram denoted as $H^{I\left[l_{s}, l_{f}\right]}$, the probability density function of input image denoted as $P^{I\left[l_{s}, l_{f}\right]}$ and the probability distribution function of input image denoted as $C^{I\left[l_{s}, l_{f}\right]}$. The equations are presented below:

$$
\begin{aligned}
& P_{l}^{I\left[l_{s}, l_{f}\right]}=\frac{H_{l}^{I}}{\sum_{i=l_{s}}^{l_{f}} H_{i}^{I}} \\
& C_{l}^{I\left[l_{s}, l_{f}\right]}=\frac{1}{\sum_{i=l_{s}}^{l_{f}} H_{i}^{I}} \sum_{i=l_{s}}^{l} H_{i}^{I}=\sum_{i=l_{s}}^{l} P_{i}^{I\left[l_{s}, l_{f}\right]}
\end{aligned}
$$

1. Generate the uniform histogram of output image denoted as $H^{O\left[l_{s}, l_{f}\right]}$. Where, every level $l$ that satisfies $0 \leq l s \leq l \leq l f \leq L-1$, has the equal sum of pixels. The uniform histogram is generated using the following formula:
$H_{l}^{O\left[l_{s}, l_{f}\right]}=\frac{1}{l_{f}-l_{s}+1} \sum_{l=l_{s}}^{l_{f}} H_{l}^{I\left[l_{s}, l_{f}\right]}$

2. Calculate the uniform probability distribution function using the subsequent equation:

$P_{l}^{o\left[l_{s}, l_{f}\right]}=\frac{1}{l_{f}-l_{s}+1}$

3. Then, compute the cumulative density function denoted by $C^{O\left[l_{s}, l_{f}\right]}$, which is defined as:

$$
C_{l}^{O\left[l_{s}, l_{f}\right]}=\frac{1}{\sum_{i=l_{s}}^{l_{f}} H_{i}^{\left[\left[l_{s}, l_{f}\right]\right.}} \sum_{i=l_{s}}^{l} H_{i}^{I\left[l_{s}, l_{f}\right]}=\sum_{i=l_{s}}^{l} P_{i}^{I\left[l_{s}, l_{f}\right]}=\frac{i-l_{s}+1}{\left(l_{f}-l_{s}+1\right)}
$$

4. Minimizing the difference between $C_{l^{\prime}}^{O\left[l_{s}, l_{f}\right]}$ and $C_{l^{\prime}}^{I\left[l_{s}, l_{f}\right]}$

to obtain the output level $(l ')$.

The resulted image would have a better contrast due to the global enhancement provided by the histogram equalization.

\section{Laplacian Sharpening Filter}

Laplacian filter is widely used as an image sharpening (deblurring) procedure. It consists of a $3 \times 3$ kernel that have three types, $-4,-8$ and 9 mid-pixel kernels as shown in Figure 3.

\begin{tabular}{|c|c|c|}
\hline 0 & 1 & 0 \\
\hline 1 & -4 & 1 \\
\hline 0 & 1 & 0 \\
\hline
\end{tabular}

\begin{tabular}{|c|c|c|}
\hline 1 & 1 & 1 \\
\hline 1 & -8 & 1 \\
\hline 1 & 1 & 1 \\
\hline
\end{tabular}

\begin{tabular}{|c|c|c|}
\hline-1 & -1 & -1 \\
\hline-1 & 9 & -1 \\
\hline-1 & -1 & -1 \\
\hline
\end{tabular}

Figure 3. The Laplacian kernels

To implement the (-4) and (-8) mid-pixel kernels, the following equation is used:

$$
F=I ?[I \otimes L K]
$$

Where, (F) is the sharpened image, (I) is the blurry image, (LK) is Laplacian kernel, and $(\otimes)$ is a convolution process. As well, to implement the (9) mid-pixel kernel, the following equation is used:

$$
F=I \otimes L K
$$

The amount of sharpening relies on the type of kernel used, for example, the (-8) and (9) mid-pixel kernels sharpen more than the (-4) mid-pixel kernel [21].

\section{Region Growing}

The region growing method is used as a segmentation technique. Its basic idea is merging the similar pixels into a region. Starting from seed point, the region grows by 
expanding adjacent pixels that satisfies the similar criteria and merge them. The newly merged pixels become the new seed point [16]. This method is suitable for MRI images because it contains object borders that are difficult to identify. Likewise, Similarity is a crucial property in region growing method, where it can be based on model, shape or intensity [17]. The basic condition used to accept the similarity of regions is described as:

$$
H\left(R_{i}\right)=T R U E \quad i=1,2,3, \ldots, p
$$

Where, $(P)$ is the quantity of regions in an image, $H\left(R_{i}\right)$ is an evaluation function to identify similarity of the region $\left(R_{i}\right)$. Then, an IF-ELSE statement is derived to decide the regions that have similar criteria or less than a predefined threshold (T) as the following:

$$
H\left(R_{i}\right)= \begin{cases}T R U E & \text { if }=|f(j, k)-f(m, n)|<T \\ F A L S E & \text { Otherwise }\end{cases}
$$

The threshold $(T)$ is an important value that affects the performance of the region growing by producing holes since the pixels cannot be merged due to the unsuitable threshold value. This article adopted a model that combines the gradients and variances within the boundary curve [18] since it can represent the uniformity of the region and the smoothness of the boundary. The calculation of the threshold $(T)$ is based on gradients and variances [21]. The region growing method is implemented as the following:

1. Let $f(x, y, a, m)$ be the fitting function of region of the input image, where:

$f(x, y, a, m)=\sum_{i+j \leq m} a_{i j} x^{i} y^{j}$

2. Calculate the error value of a pixel with respect to the region using the subsequent equation. Moreover, the small error value can be used to determine pixels $g(x, y)$ should be grouped into the same region.

$$
E(R, a, m)=\sum_{(x, y) \in R} g(x, y)-f(x, y, a, m)^{2}
$$

3. Divide the image into initial seed regions $R_{i}^{(0)}$.

4. On the seed regions, evaluate whether the model is fitted or not. If $\mathrm{E}\left(R_{i}^{(0)}, \mathrm{a}, \mathrm{m}\right)<$ predefined value, accept $R_{i}^{(0)}$ and its model; if not discard $R_{i}^{(0)}$.

5. Find all points in each region that are well-suited to the seed-point by taking into account the neighbor pixels of current region using the following:

$$
C_{i}^{(k)}=\left[\begin{array}{l}
\frac{(x, y)}{(g(x, y)-f(x, y, a, m))^{2}} \\
\text { and }(x, y) \text { is a } 4 \text { - neighbor of } R_{i}^{(k)}
\end{array}\right]<\mathcal{E}
$$

6. If $C_{i}^{(k)} \geq \varepsilon$, then $\mathrm{m}=\mathrm{m}+1$. If $\mathrm{m}>\mathrm{M}$, then $R_{i}^{(k)}$ should not grow further; Else, go to step three (3).

7. Unite the new region $R_{i}^{(k)}$ to region $C_{i}^{(k)}$,

$R_{i}^{(k+1)}=R_{i}^{(k)} \cup C_{i}^{(k)}$

Then, evaluate the current model again with $R_{i}^{(k+1)}$, by calculating $E\left(R_{i}^{(k+1)}, a, m\right)$.

8. Calculate the error using the subsequent equation. If $\rho^{(k)}<T$, update $\mathrm{T}=\mathrm{h}$ and move to step three (3). Otherwise, $\mathrm{m}=\mathrm{m}+1$; Likewise, check whether $\mathrm{m}>\mathrm{M}$, if true then stops the region growing.

$$
P(k)=E\left(R_{i}^{(k+1)}, a, m\right)-E\left(R_{i}^{(k+1)}, a, m\right)
$$

9. Evaluate the current region with the new model $f(x, y, a, m)$. If the error of the new model is less than the previous model, then allow the new model and move to step three (3). If the error is greater than its previous value, then stop the region growing.

This article adopts the optimal dynamic threshold, which taking into account the pixels on the edges and regions. The method is described below:

1. Assume a defined number of iterations to calculate the threshold as $t$. The coarse contours denoted by $s=\left\{s_{k}\right\}$, where $0<k<t$.

2. If there any (ni) gray-level, then, the gradient of each gray-level is calculated as $g_{s_{k}}$ and the mean gradient as ${ }^{s_{k}}$ using the following equations:

$$
\begin{gathered}
g_{s_{k}}=\frac{\sum_{p=1}^{n_{k}} g_{p}}{n_{k}} \\
\boldsymbol{G}_{s_{k}}=\frac{1}{\boldsymbol{g}_{s_{k}}}
\end{gathered}
$$

Where, the mean variance denoted by $\sigma_{s_{k}}^{2}$ is described as follows:

$$
\mu_{s_{k}}=\frac{\sum_{i=0}^{L-1}\left(i n_{i}\right)}{\sum_{i=0}^{L-1}\left(n_{i}\right)}
$$




$$
\sigma_{s_{k}}^{2}=\frac{\sum_{i=0}^{L-1}\left(i-\mu_{s_{k}}\right)^{2} n_{i}}{\sum_{i=0}^{L-1}\left(n_{i}\right)}
$$

3. Based on the mean gradient and the variance, the optimal threshold value (h) is calculated using the subsequent equations:

$$
h s k=a G_{s k}+\beta \sigma_{s k}(\alpha, \beta \text { are weight coefficient })
$$

$$
h=\arg \min h_{s k}
$$

Finally, The coefficients $\alpha$ and $\beta$ are obtained as the following:

$$
\left\{\begin{array}{l}
\alpha: \beta=\frac{\delta}{\pi}: 2 \pi \\
\alpha+\beta=1
\end{array}\right.
$$

\section{EXPERIMENTAL RESULTS}

In this article, three restoration and enhancement procedures are implemented to improve the quality of the processed MRI images prior to the segmentation process. Firstly, a denoising algorithm is applied to reduce the noise from the image. Secondly, a contrast enhancement technique is applied to provide the normal image intensity. Thirdly, a sharpening filter is applied to decrease the image blur. Then, the seed point is selected and the tumor is segmented. Figure 4 illustrates the conducted experiments.
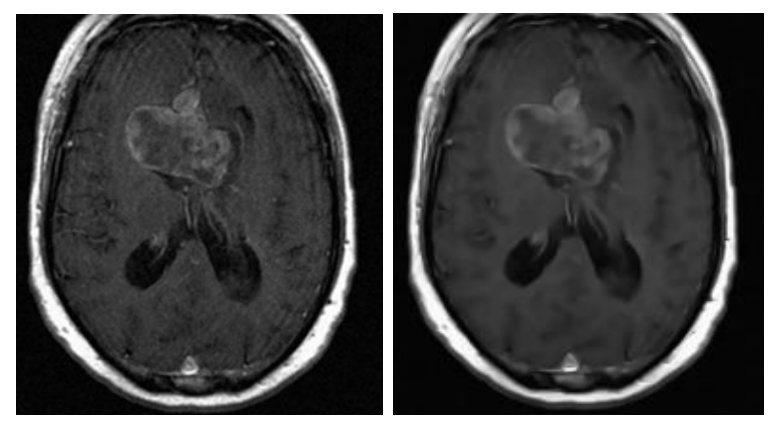

(a)

(b)
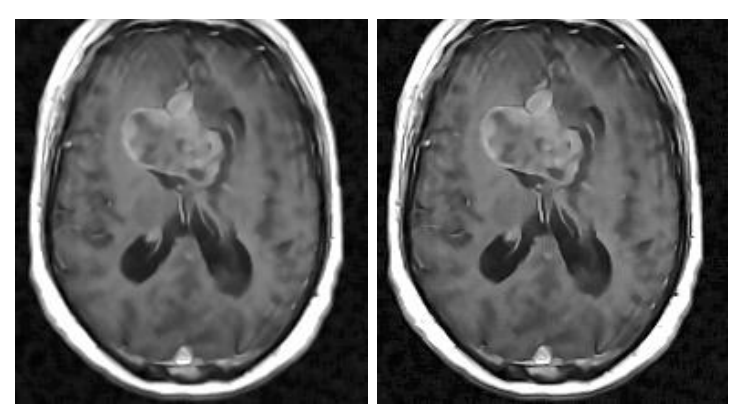

(c)

(d)
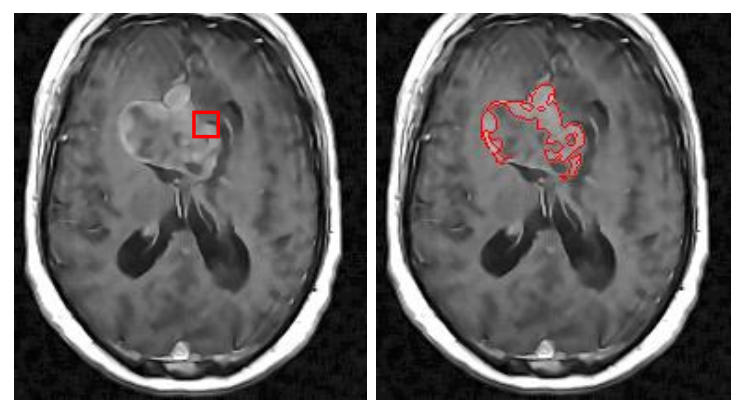

(e)

(f)

Figure 4. a) Original MRI image; b) Denoised by NL-Means; c) Enhanced by Histogram Equalization; d) Sharpened by Laplacian; e) Seed point Selection; f) Segmented by Reign Growing

\section{DISCUSSION}

Typically, common image degradations such as noise, blur and low contrast affects the performance of segmentation by causing errors in the segmented areas. Firstly, noise which is the unwanted image component alters the intensities of the pixels causing errors in the segmentation process as shown in Figure 5. Secondly, the low contrast artifact may affect the segmentation process by reducing the volume of the detected tumor since some parts of the tumor tissue own the same intensities as the healthy tissue. Figure 6 illustrates the segmentation process before and after enhancing the contrast where, the amount of the segmented tumor increased after enhancing the contrast. Thirdly, the blurring artifact may cause a faulty segmentation process because the blur hides information that indeed exists in an image and smooth the image edges as well as its fine details. Figure 7 illustrates the segmentation process before and after deblurring the MRI image where, the deblurred image gave more precise segmentation results. 

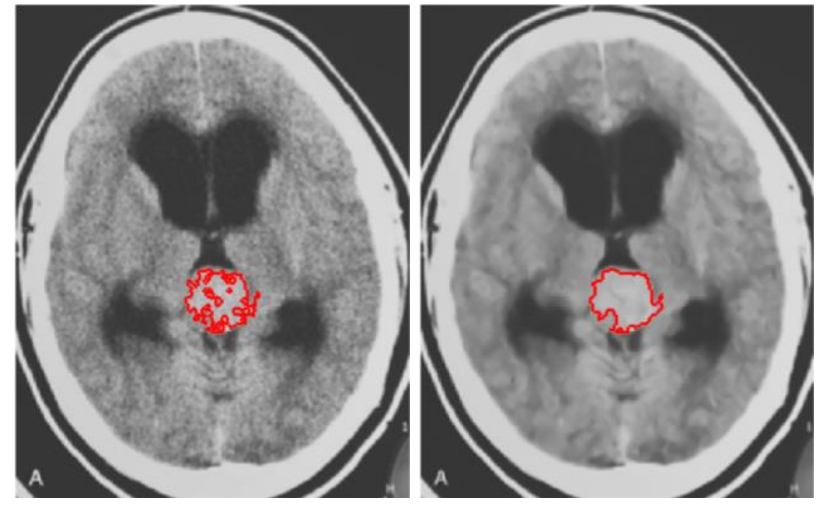

(a)

(b)

Figure 5. a) The segmentation of a noisy image; b) the segmentation of a denoised image
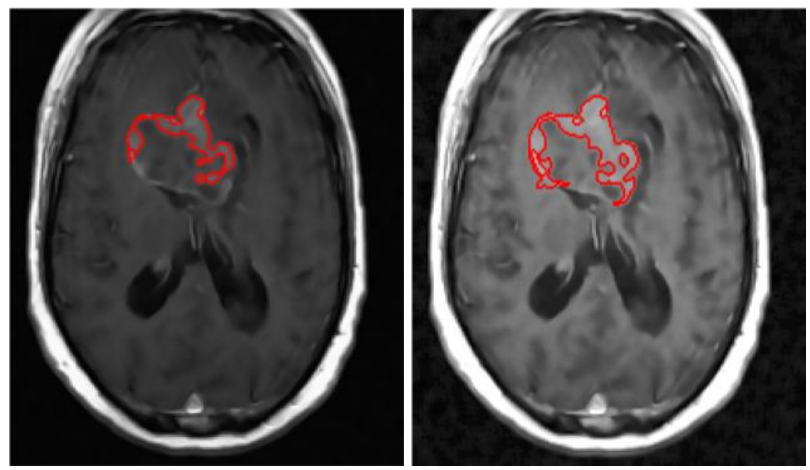

(a)

(b)

Figure 6. a) The segmentation of a low-contrast image; b) the segmentation of a contrast enhanced image
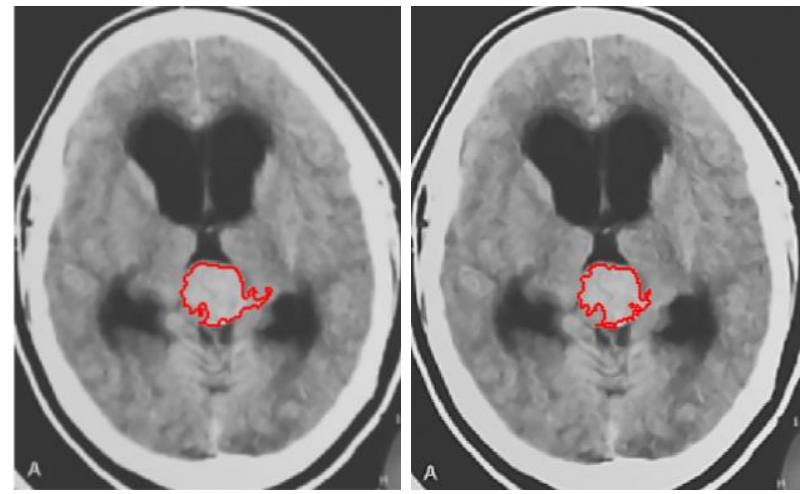

Figure 7. a) The segmentation of a blurry image; b) the segmentation of a deblurred image

Finally, reducing image degradations using proper image processing algorithms is an important step to obtain more accurate segmentation results. Likewise, various algorithms exist which are specialized in removing a specific type of degradation. Then, proper algorithms should be used with the correct degradation.

\section{CONCLUSION}

This article provides a better segmentation process for MRI images through the use of proper image enhancement and restoration techniques. Many images that are obtained from different databases used to show the efficiency of the proposed scheme. Combining different techniques is very important because each algorithm has its own specific characteristics to handle a particular problem. Finally, the resulted images hold not only a better quality but a more precise tumor segmentation procedure.

\section{REFERENCES}

[1] Chugh, S., \& Anand, S. M. (2012). Semi-Automated Tumor Segmentation from MRI Images Using Local Statistics Based Adaptive Region Growing. International Journal of Information and Electronics Engineering, 2(1).

[2] Clark, M. C., Hall, L. O., Goldgof, D. B., Velthuizen, R., Murtagh, F. R., \& Silbiger, M. S. (1998). Automatic tumor segmentation using knowledgebased techniques. Medical Imaging, IEEE Transactions on, 17(2), 187-201.

[3] Zhang, J., Zheng, J., \& Cai, J. (2010, June). A diffusion approach to seeded image segmentation. In Computer Vision and Pattern Recognition (CVPR), 2010 IEEE Conference on (pp. 2125-2132). IEEE.

[4] Pizurica, A., Wink, A. M., Vansteenkiste, E., Philips, W., \& Roerdink, B. J. (2006). A review of wavelet denoising in MRI and ultrasound brain imaging. Current medical imaging reviews, 2(2), 247-260.

[5] Noll, D. C., Pauly, J. M., Meyer, C. H., Nishimura, D. G., \& Macovskj, A. (1992). Deblurring for non-2D Fourier transform magnetic resonance imaging. Magnetic Resonance in Medicine, 25(2), 319-333.

[6] Al-Manea, A., \& El-Zaart, A. (2007, January). Contrast enhancement of MRI images. In 3rd Kuala Lumpur International Conference on Biomedical Engineering 2006 (pp. 255-258). Springer Berlin Heidelberg.

[7] Rajendran, A., \& Dhanasekaran, R. (2012). Brain Tumor Segmentation on MRI Brain Images with Fuzzy Clustering and GVF Snake Model. With Emphasis on the Integration of Three Technologies, 495.

[8] Zhang, J., Ma, K. K., Er, M. H., \& Chong, V. (2004). Tumor segmentation from magnetic resonance imaging by learning via one-class support vector machine. In International Workshop on Advanced Image Technology (IWAIT'04) (pp. 207-211).

[9] M. Kumar and K. K. Mehta, "A Texture based Tumor detection and automatic Segmentation using Seeded Region Growing Method", International Journal of Computer Technology and Applications, 2 (4), 2013. 
[10] Faisal, A., Parveen, S., Badsha, S., Sarwar, H., \& Reza, A. W. (2013). Computer Assisted Diagnostic System in Tumor Radiography. Journal of medical systems, 37(3).

[11] Afifi, A. (2012). A Hybrid Technique Based on Combining Fuzzy K-means Clustering and Region Growing for Improving Gray Matter and White Matter Segmentation. International Journal of Advanced Computer Science and Applications, 3(7).

[12] Efros, A. A., \& Leung, T. K. (1999). Texture synthesis by non-parametric sampling. In Computer Vision, 1999. The Proceedings of the Seventh IEEE International Conference on (Vol. 2, pp. 1033-1038). IEEE.

[13] Buades, A., Coll, B., \& Morel, J. M. (2004). On image denoising methods. CMLA Preprint, 5.

[14] Buades, A., Coll, B., \& Morel, J. M. (2005, June). A non-local algorithm for image denoising. In Computer Vision and Pattern Recognition, 2005. CVPR 2005. IEEE Computer Society Conference on (Vol. 2, pp. 60-65).

[15] Mohanta, K., \& Khanaa, V. (2013). An Efficient Contrast Enhancement of Medical X-Ray ImagesAdaptive Region Growing Approach. International Journal of Engineering and Computer Science, 2(2).

[16] Umamaheswari, J., \& Radhamani, G. (2012). An Enhanced Approach for Medical Brain Image Enhancement. Journal of Computer Science, 8(8), 1329.

[17] Kim, Y. T. (1997). Contrast enhancement using brightness preserving bi-histogram equalization. Consumer Electronics, IEEE Transactions on, 43(1), 1-8.

[18] Sapna Josephus, C., \& Remya, S. (2012). Enhancement Techniques for Local Content Preservation and Contrast Improvement in Images.

[19] Goyal, S. (2011). " Region Based Contrast Limited Adaptive HE with Additive Gradient for Contrast Enhancement of Medical Images (MRI)". International Journal of Soft Computing and Engineering (IJSCE), 1(4).

[20] Menotti, D., Najman, L., Facon, J., \& De Araujo, A. A. (2007). Multi-histogram equalization methods for contrast enhancement and brightness preserving. Consumer Electronics, IEEE Transactions on, 53(3).

[21] Al-Ameen, Z., Sulong, G. \& Johar, M. G. M. (2012). A comprehensive study on fast image deblurring techniques. ", International Journal of Advanced Science and Technology (IJAST), 44, 1-10.

[22] Deng, W., Xiao, W., Deng, H., \& Liu, J. (2010, October). MRI brain tumor segmentation with region growing method based on the gradients and variances along and inside of the boundary curve. In Biomedical Engineering and Informatics (BMEI), 2010 3rd International Conference on (Vol. 1, pp. 393-396). IEEE.

Ahmed Basil Al-Othman was born in Iraq in 1981. He has received his B.Sc. in computer science from AlHadba University College in 2005. In 2011 he received his M.Sc. degree in Computer Science from Universiti Teknology Malaysia (UTM). His area of research interest is medical image processing, image enhancement, pattern and face recognition, segmentation, and GIS.

Zohair Al-Ameen was born in the United Kingdom in 1985, obtained his B.Sc. degree in Computer Science from the University of Mosul - IRAQ in 2008. In 2011 he obtained his M.Sc. in Computer Science from Universiti Teknologi Malaysia (UTM). His research interests include Image and Video Processing, Image Restoration, Image Enhancement, Medical Imaging, Segmentation, Optical Characters Recognition, Motion Detection, and Pattern Recognition. Currently he is a full time Ph.D. student at the faculty of computing, Universiti Teknologi Malaysia (UTM).

Ghazali Bin Sulong received his BSc degree in statistic from National University of Malaysia, in 1979, and MSc and $\mathrm{PhD}$ in computing from the University of Wales, Cardiff, United Kingdom, in 1982 and 1989, respectively. $\mathrm{He}$ is currently professor at Faculty of Computing, Universiti Teknologi Malaysia. His research interest includes Biometric - fingerprint identification, face recognition, iris verification, ear recognition, handwritten recognition, and writer identification; object recognition; image enhancement and restoration; medical imaging; human activities recognition; data hiding - digital watermarking, steganography and image encryption; image fusion; image mining; digital image forensics; object detection, segmentation and tracking.

How to cite this paper: Ahmed Basil Al-Othman,Zohair Al-Ameen,Ghazali Bin Sulong,"Improving the MRI Tumor Segmentation Process Using Appropriate Image Processing Techniques", IJIGSP, vol.6, no.2, pp.23-29, 2014.DOI: 10.5815/ijigsp.2014.02.03 